\title{
Microstructure and mechanical properties of grains of silica nanofluids dried in an acoustic levitator
}

\author{
Rosa Mondragon $^{1}$, J. Enrique Julia ${ }^{2}$, Antonio Barba ${ }^{1}$, Juan Carlos Jarque ${ }^{1, *}$ \\ ${ }^{1}$ Instituto de Tecnología Cerámica. Universitat Jaume I. \\ Campus de Riu Sec. 12071-Castellón de la Plana. Spain \\ 2 Departamento de Ingeniería Mecánica y Construcción. Universitat Jaume I. \\ Campus de Riu Sec. 12071-Castellón de la Plana. Spain
}

* Corresponding author. E-mail: juancarlos.jarque@itc.uji.es. Tel: +34 964342424. 


\begin{abstract}
The microstructure and mechanical properties of spray-dried grains are key factors in many applications. In this work single droplets of silica nanofluids were dried in an acoustic levitator under different experimental conditions of solid mass fraction, $p H$ value, salt concentration, drying temperature and initial droplet volume. ANOVA method was used to determine their influence on the final grain diameter, the shell thickness and the mechanical strength. The solid content and the droplet volume are the variables that exert an influence on these three properties. In addition, the mechanical strength is influenced by the $p H$ value. The maximum packing fraction of the particles inside the shell was obtained by modelling the viscosity data with the Quemada equation. The packing fraction was then used to calculate the shell thickness. The internal microstructure of the grains was observed by SEM and the shell thickness was measured. Experimental and calculated results show good agreement.
\end{abstract}

Key words: Drying, Nanofluids, Microstructure, Strength, Acoustic levitator 


\section{Introduction}

In many industrial applications such as ceramics, food products, detergents and pharmaceuticals, spray drying is used to produce powders with different characteristics. This process causes the solvent of the droplets to evaporate, leading to the formation of dried grains [1]. Depending on the final application of these grains and their later handling and processing, different microstructures may be required. This makes it of great importance to know how all the variables involved in the drying process affect the crust formation, the packing of the particles, the hollowness of the final grain, and so forth. A good knowledge and control of these parameters can lead to the production of grains with suitable characteristics for each application.

The drying behaviour of a liquid-solid suspension droplet can be divided into two stages [1-3]. In the first stage, also known as the constant rate period, particles move towards the inner part of the droplet to minimize its surface energy $[4,5]$. The surface energy of the liquid-vapour interface is lower than that of the solid-vapour one and so the particles move, thus allowing the droplet surface to be completely wetted and the liquid to evaporate at the droplet surface. When the critical moisture content is reached, the entire droplet surface can no longer be kept saturated by moisture migration and the second drying period begins. In this period, called the falling rate period, a shell is formed on the droplet surface and the evaporation occurs through the pores of the shell.

When the second drying period starts, the liquid menisci formed between particles create a capillary force that makes them approach each other and keep together [6]. Moreover, the latent heat of water evaporation has a high value. When a small amount of water evaporates, a strong flux of heat is transmitted from the surface of the droplet into the gas stream and local temperature gradients are created on the droplet surface. This causes the thermophoretic displacement of the particles towards the surface of the droplet $[7,8]$. As a result, the shell thickness increases due to the progressive incorporation of particles into the shell.

The packing fraction of the particles in the shell is constant and closer to the maximum packing that particles can achieve, i.e. random close packing $[6,9]$. In particulate systems, two packings are possible: random close packing (RCP) represents the densest packing state that uniform spheres can achieve when randomly packed, while random loose packing (RLP) refers to the least dense packing that can resist an external load [10-12]. For hard, monodispersed, spherical particles with a micrometric size the final volume fractions 
achieved at each packing state are established at $\phi_{R C P}=0.64$ and $\phi_{R L P}=0.56$ for RCP and RLP respectively. However, these values depend on the particle size and the interparticle forces present in the system.

For particles in the nanometric size range, the Van der Waals cohesive forces become dominant. This force restricts the relative movement of the particles, thereby resulting in the formation of agglomerates that affect the packing of particles. Hence, the volume fraction for RCP depends on the Hamaker constant and particle size [11, 13, 14]. Moreover, in nanofluids, capillary forces are present at the final stage of the packing process and these affect the packing [15-17]. In general, cohesive forces hinder the rearrangement of the particles and lead to lower packing fractions.

The maximum packing fraction that the nanoparticle system can achieve (RCP) corresponds to the fluidity limit, $\phi_{m}$. Below this value the nanofluid behaves like a liquid, while above the fluidity limit the nanofluid has the properties of a solid. The graphical representation of viscosity versus the volume fraction makes it possible to obtain the fluidity limit, which is the concentration at which the viscosity takes an infinite value. Krieger and Dougherty, and Quemada $[18,19]$ proposed equations to model the viscosity of numerous suspensions with particles of different natures, which allows the fluidity limit and hence the $\mathrm{RCP}$, to be determined for each particulate system.

The degree of hollowness of the grains depends on the final grain diameter and the packing of the particles. By modifying the properties of the suspension, the maximum packing fraction of the particles can also be modified. This packing influences the volume occupied by the particles in the shell and its thickness, which in turn determines the degree of hollowness of the dried grains for a constant final diameter. Otherwise, for a particular system with a constant packing fraction, the shell thickness depends on the final diameter. This diameter varies according to the capacity of the particles to diffuse inside the droplet, thus modifying the instant in which the collapse of particles takes place and the second drying period starts. The strength of drying is quantitatively represented by the Peclet number, $P e$, which characterizes the relative importance of the time scale for diffusion $\left(r_{D}{ }^{2} / D_{S}\right)$ with respect to that of the convective drying time $(t)[8,9,20]$ :

$P e=\frac{r_{D}^{2} / D_{S}}{t}$ 
where $r_{D}$ is the droplet radius, $t$ is the drying time and $D_{S}$ is the diffusion coefficient, which can be calculated from the Einstein-Stokes relation:

$$
D_{S}=\frac{k_{B} \cdot T_{D}}{6 \cdot \pi \cdot \eta \cdot r_{p}}
$$

where $k_{B}$ is the Boltzman constant, $T_{D}$ is the droplet temperature, $\eta$ is the viscosity of the nanofluid and $r_{p}$ is the radius of the particles diffusing inside the droplet.

If the Peclet number $P e>>1$, the drying is faster than the rate of diffusion of the particles and the time needed for the liquid to evaporate is shorter than the time required to homogenize the droplet. In this case the drying is said to be fast and the collapse takes place earlier, thus leading to the formation of a hollow or crumpled grain. In contrast, if $P e<<1$, the drying is regarded as a slow process, particles have time to diffuse towards the core of the droplets and they shrink isotropically, resulting in compact grains with smaller diameters.

In this work, single droplets of silica nanofluids were dried in an acoustic levitator under different experimental conditions, in accordance with a Design of Experiments (DoE). The ANOVA method was used to determine the influence of the drying conditions on the characteristics and properties of the grains obtained, i.e. final grain diameter, shell thickness and mechanical strength. The fluidity limit and the maximum packing fraction were obtained for this system using the Quemada equation to model the viscosity data. The packing fraction and the final diameter were used to calculate the shell thickness and compare it with the experimentally measured thickness.

\section{Experimental set-up and measurement techniques}

\subsection{Rheological measurements}

The viscosity of the nanofluids was obtained by conducting tests under steady-state conditions using a Bohlin CVO-120 rheometer (Malvern Instruments Ltd., UK). A double gap (DG 40/50) device composed of two concentric cylinders suitable for low viscosity suspensions was used. The sample is introduced in the gap between the inner cylinder $($ diameter $=40 \mathrm{~mm})$ and the outer cylinder $($ diameter $=50 \mathrm{~mm})$. A cylindrical device with a diameter of $45.46 \mathrm{~mm}$ is submerged into the sample, thereby creating the double gap. Before each test, a pre-treatment, in which the samples are submitted to a constant shear stress, was applied to the nanofluids for 30 seconds to ensure similar starting conditions for all of them. 


\subsection{Drying of droplets}

Droplets were dried in an acoustic levitator. This equipment was previously used to study the drying behaviour of single droplets of different materials under a variety of experimental conditions [21-28]. Levitator tubes offer some advantages over conventional methods, since there is no mechanical contact with the droplet and this makes it possible to work with almost any liquid over a wide range of diameters of droplets (from $0.1 \mathrm{~mm}$ to several $\mathrm{mm}$ ) [29]. The experimental set-up and the operational conditions were described in detail in a previous work [27]. An acoustic levitator consisting of an ultrasonic $58 \mathrm{kHz}$ horn and a concave reflector (tec5 AG Sensorik und Systemtechnik) modified in order to work at high temperature conditions of up to $150^{\circ} \mathrm{C}$ was used. The flow rate of the air stream was set to $0.5 \mathrm{l} / \mathrm{min}$ and used to ensure constant drying conditions around the droplet and to ventilate the acoustically-induced vortex system formed around the droplet from liquid vapour. The process was recorded and the images were processed with Matlab to gather the desired information.

The sound pressure level of the acoustic stream must be held at a constant and appropriate value. To levitate a drop, the ultrasound has to be strongly enough to overcome gravity force. However, if the sound pressure level is too high the drop disintegrates into small droplets, since then the capillary forces become weak to keep the drop intact [41]. Therefore, the intensity of the acoustic stream is established at a value so that the capillary and Van der Waals forces that keep particles together are not influence by the levitation process. The acoustic streaming in the gas provides a convective mechanism much stronger than the natural convection and dominates the evaporation process thus affecting the drying rate but not the motion of the particles inside the droplet [42].

\subsection{Grain properties}

The internal microstructure of the grains obtained was observed by means of Scattering Electron Microscopy using an FEI Quanta 200F microscope. The grains were cut and coated with platinum in order to obtain cross-sections and to observe the internal shell formed during the drying process. Images were taken of the shell and the arrangement of the nanoparticles inside it.

Moreover, in order to obtain the mechanical strength of the granules, diametrical compression tests of single granules were performed. The equipment used (Instron 5889) has a special compression cell (maximum load of $10 \mathrm{~N}$ ) that allows individual grain tests to be 
performed. Typical force-displacement curves were acquired for each experimental condition and these were then used to obtain the fracture load of the grains. In the curves it can be seen how, when the compression device comes into contact with the granule, the force increases until the breaking point.

\section{Suspensions and experimental matrix}

All the experiments were carried out with silica-water nanofluids. In this work commercial fumed silica provided by Degussa was used. The silica chosen was an Aerosil 200 consisting in amorphous hydrophilic silica nanoparticles with primary units of $12 \mathrm{~nm}$ and a density of $2200 \mathrm{~kg} / \mathrm{m}^{3}$ according to the manufacturer. Nanofluids with different particle concentrations were prepared by adding distilled water to the defined amounts of nanoparticles. In this method, known as the two-step method, the nanoparticles are purchased as a dry powder and then dispersed in the liquid medium. The dispersion was performed using an ultrasonic probe (UP400s from Hielscher Company), which has been proven to be the most effective dispersion system [30]. The procedure that allows a good dispersion to be achieved was established by the authors in a previous work [31]. Initially, the mixture of nanoparticles with the water is submitted to a sonication treatment for 3 minutes. After this, the $p H$ of the nanofluid is modified by adding small amounts of $\mathrm{HCl}$ or $\mathrm{NaOH}$ solutions $(2.75 \mathrm{M})$. Finally, to ensure correct dispersion of all the components, the nanofluids are submitted to a second sonication treatment for 2 minutes. In the same previous study, the isoelectric point (IEP) for this system was experimentally determined from the zeta potential curves and a $p H=2$ value was obtained.

To gather experimental information about the internal microstructure of the grains and their mechanical strength at different experimental conditions a DoE was planned. In this case, a matrix based on a fractional factorial design, $2^{k-1}$, was performed. Five variables including the solid mass fraction (expressed as $\mathrm{kg}$ of solid per $\mathrm{kg}$ of suspension): $0.02 \mathrm{w} / \mathrm{w}<$ $Y_{S}<0.20 \mathrm{w} / \mathrm{w} ; \mathrm{pH}$ of nanofluids: $2<p H<10$; salt concentration; $0 \mathrm{M}<[\mathrm{NaCl}]<0.05 \mathrm{M}$; ambient air temperature: $80^{\circ} \mathrm{C}<T<120^{\circ} \mathrm{C}$; and initial droplet volume: $0.3 \mu \mathrm{l}<V_{0}<0.8 \mu \mathrm{l}$ were chosen to study their effect on the packing of particles and physical properties. A total number of 16 tests were carried out for the five input variables.

\section{Results and discussion}

\subsection{ANOVA analysis}


Table 1 shows the experimental matrix and the conditions under which the drying tests were carried out. For each test performed, the final grain diameter $\left(d_{G}\right)$ was obtained from the image processing of the videos that were recorded. The shell thickness (s) was measured from the SEM images. Finally, grains produced in all the drying experiments were submitted to mechanical strength tests. For each experimental condition, the maximum force resisted by the grain before breaking $(F)$ was obtained. The drying and mechanical tests were repeated 8 times to obtain mean values for the parameters analysed and to evaluate the reproducibility of the experimental set-up. The shell thickness was established by taking the mean of 5 values measured at different regions of the cut grains. Errors of less than $10 \%$ were obtained for all the properties measured.

Table 1 shows the results obtained for each output variable mentioned above. All the results were analysed using the ANOVA method and the significance of each effect on each output variable was obtained. This method determines the degree of significance from the probability of the Fisher distribution. It is said that an input variable has a statistically significant effect on the output variable at the $95 \%$ confidence level when the probability of the F-distribution is less than 0.05 . Table 2 shows the values obtained for the probability of the F-distribution for each input variable and measured property. In the sections that follow, all the results and the analysis of variables are shown.

\section{Grain diameter $\left(d_{G}\right)$}

In Table 2 it can be seen that the only two input variables that influence the final grain diameter are the solid content and the initial droplet volume. In Figure 1 a) it can be observed that both effects are positive. This means that an increase in the value of the input variable leads to an increase in the output one.

When the solid content is increased, there are more particles inside the droplet and the final volume of solids is higher, thus producing bigger grains. Moreover, at a constant solid content, bigger droplets contain higher amounts of solids, which finally also produce bigger grains.

\section{$\underline{\text { Shell thickness }(s)}$}

In Table 2 it can be seen that the input variables that influence the shell thickness are the solid content and the initial droplet volume. Not only their single effect but also the cross effect are significant for this output variable. 
In Figure 1 b) it can be observed that both single effects are positive. An increase in the solid content leads to a higher amount of solid inside the droplet when the shell is formed. As a result, the thickness of the shell is bigger. Furthermore, bigger droplets, containing higher amounts of solid, produce thicker shells [5]. Actually, from the cross effect it can be seen that when the solid content is very low, the influence of the droplet volume on the shell thickness is negligible. However, it is at high solid contents when the influence of the droplet volume becomes important. The influence of the solid content is enhanced when it is high due to the larger number of particles inside the droplet.

\section{Mechanical strength $(F)$}

The mechanical strength of the grains is of great importance. Often they must resist handling, as well as later treatments and stages, and this property can influence the quality of the final product. The mechanical strength is related to the microstructure of the grains. In general, dense solid granules are more resistant while hollow ones break more easily when an external load is applied.

In Table 2 it can be seen that the input variables that influence the force resisted by the grain are the solid content, the droplet volume and the $p H$ of the nanofluid. Not only their single effect but also the cross effect of the solid content with the other two variables are significant.

In Figure $1 \mathrm{c}$ ) it can be observed that the single effects are positive. The influence of the solid content and the droplet volume is related to the effect of these two variables on the shell thickness. As mentioned before, an increase in the solid content or the droplet volume leads to thicker shells. This makes the grains denser and, as a result, the force the grains can resist is also increased by raising the values of these two parameters. In the same way as the shell thickness, this effect is more important for high solid contents.

The effect of the $\mathrm{pH}$ of the nanofluid also depends on the solid content. For low solid contents, the mechanical strength is not dependent on the $p H$ value. However, for high solid contents, the mechanical strength increases with the $p H$ value. This trend is related to the evolution of the solubility of silica particles in water $[32,33]$. The dissolution of silica in water is, in effect, a depolymerization through hydrolysis and the "solubility" is the concentration of $\mathrm{Si}(\mathrm{OH})_{4}$ and other silicate species. For silica, the hydroxyl ion is a unique catalyst and the solubility of silica in water can be increased by making the solution more alkaline. As a result, at high $p H$ values, the solubility/dissolution rate increases and necks are 
formed between particles, thereby increasing the strength of the bond. The influence of the $p H$ value is more important for high solid contents because of the large number of particles involved in the process.

The influence of the $p H$ value and the shell thickness (which in turn depends on the solid content and the droplet volume) can be observed by plotting the force resisted by the grain versus the shell thickness. In Figure 2, the influence of the variables analysed before can be observed and confirmed. First, as the shell thickness increases, the grain becomes more compact and denser, and consequently it can resist a higher external load. Secondly, for a high shell thickness, the mechanical strength increases with the $p H$.

\subsection{Particle packing in the shell}

In order to calculate the fluidity limits and the maximum packing fraction of the particles, the Quemada equation was used to model the viscosity data at different solid contents for the two $\mathrm{pH}$ values studied.

$\eta_{r}=\frac{\eta}{\eta_{F}}=\left(1-\frac{\phi}{\phi_{m}}\right)^{-2}$

where $\eta_{r}$ is the relative viscosity, $\eta$, is the viscosity of the nanofluid, $\eta_{F}$ is the viscosity of the base fluid and $\phi_{m}$ is the fluidity limit.

Additional nanofluids were prepared at volume fractions ranging from 0.002 to 0.132 $\mathrm{m}^{3}$ of solid per $\mathrm{m}^{3}$ of suspension, which corresponds to mass fractions from 0.005 to $0.25 \mathrm{~kg}$ of solid per $\mathrm{kg}$ of suspension. A total number of 8 different nanofluids were prepared within this range of solid content to cover a wide range of viscosities, obtaining results in the nonlineal region of the curve. This number of experimental data also made it possible to fit them to the model equation.

To model the evolution of the viscosity results with the solid content, effective volume fractions are needed [34-36]. When nanoparticles are electrostatically stabilized, surface charges appear, forming an external double layer around the particles. The thickness of this layer depends on the presence of electrolytes and ions introduced into the nanofluids when adjusting the $\mathrm{pH}$ value (introduction of chloride and sodium ions with $\mathrm{HCl}$ and $\mathrm{NaOH}$ respectively). The use of effective volume fractions makes it possible to screen the effect of the electrolyte concentration on the viscosity in order to model the experimental data. The thickness of the electrical double layer or Debye length, $\kappa^{-1}$, was calculated from the electrical conductivity measurements of each nanofluid by means of equations 4 and 5: 
$\kappa^{-1}=0.215 \cdot 10^{-9} \cdot \sqrt{I}$

where $I$ is the ionic strength of the medium and is calculated from the electrical conductivity. Griffin and Jurinak [37] obtained the following empirical equation to calculate the ionic strength in suspensions with the presence of sodium and chloride ions:

$I=0.013 \cdot E C$

where $E C$ is the electrical conductivity of the suspension, which was measured directly using an EC-Meter Basic 30+ conductimeter (Crison).

With the electrical double layer, the effective volume fractions were calculated taking $6 \mathrm{~nm}$ as the value of the particle radius:

$\phi_{\text {eff }}=\phi\left(\frac{r_{p}+\kappa^{-1}}{r_{p}}\right)^{3}$

In Figure 3 experimental data are represented together with the theoretical curves corresponding to the Quemada equation. It can be seen that the viscosity results are in good agreement with the model.

As can be seen, the maximum packing fraction, $\phi_{m}$, increases as the $p H$ value decreases. For $p H=2$ (IEP) the maximum value for the fluidity limit is achieved $\left(\phi_{m}=0.33\right)$, which represents RCP. However, this value is lower than the 0.64 predicted for low hard, monodispersed spheres. In this case, Van der Waals cohesive forces are predominant. These forces restrict the relative movement of particles resulting in agglomerates with the presence of large pores and worse packings than in the case of hard spheres. When the $p H$ value is increased, particles become negatively charged and an electrical layer is formed around them. The repulsion generated between particles leads them to remain separated from each other, thus resulting in higher viscosities (secondary electroviscous effect) and less compact packings $[38,39]$. Therefore, an increase in the $p H$ value and the surface charge leads to looser packings as a consequence of the repulsion between particles.

The experiments involving drying single droplets carried out in the DoE were used to check the constant packing fraction of the particles in the shell for a particular system, regardless of the drying conditions. All tests resulted in high Peclet values ranging from $7 \cdot 10^{2}$ to $2 \cdot 10^{6}$. The Peclet numbers were calculated using equations 1 and 2, taking the radius of the agglomerates present in the nanofluid as the radius of the particles in equation 2 . Those agglomerates are the ones that diffuse, not the primary particles. The temperature of the 
droplet is the wet bulb temperature corresponding to the drying temperature for each case, and the viscosity of the nanofluid and the drying time were measured experimentally. The minimum Peclet values correspond to the lowest solid content $(0.02 \mathrm{w} / \mathrm{w})$, which provides lower viscosities $(0.0012 \mathrm{~Pa} \cdot \mathrm{s})$, a lower size of the agglomerates as measured by Dynamic Light Scattering (205 nm) and, hence, a higher diffusion coefficient $\left(1.72 \cdot 10^{-12} \mathrm{~m}^{2} / \mathrm{s}\right)$, as well as the lowest drying temperature $\left(80^{\circ} \mathrm{C}\right)$, which provides longer drying times $(91 \mathrm{~s})$. In contrast, the maximum Peclet values correspond to the highest solid content $(0.20 \mathrm{w} / \mathrm{w})$, which provides higher viscosities $(0.381 \mathrm{~Pa} \cdot \mathrm{s})$, a higher size of the agglomerates as measured by Dynamic Light Scattering (1004 nm) and, hence, a lower diffusion coefficient $\left(1.17 \cdot 10^{-15}\right.$ $\left.\mathrm{m}^{2} / \mathrm{s}\right)$, as well as the highest drying temperature $\left(120^{\circ} \mathrm{C}\right)$, which provides shorter drying times $(83 \mathrm{~s})$.

As a consequence, hollow grains were obtained in all the tests performed. The shell thickness was calculated considering a packing fraction value of $\phi_{\text {Shell }}=0.33$, which was obtained previously from the viscosity model. The packing fraction of the particles in the shell can be expressed as the ratio between the volume of solid inside the shell, $V_{S}$, and the shell volume, $V_{\text {Shell }}$ :

$\phi_{\text {Shell }}=\frac{V_{S}}{V_{\text {Shell }}}=\frac{V_{0, d} \cdot Y_{S} \cdot \rho_{0, d} / \rho_{S}}{V_{G}-V_{C}}$

where $V_{0, d}$ is the initial droplet volume, $V_{G}$ is the final grain volume, $V_{C}$ is the core volume (central pore volume), $Y_{S}$ is the initial solid mass fraction, $\rho_{S}$ is the solid density and $\rho_{0, d}$ is the initial droplet density calculated as follows:

$\frac{1}{\rho_{0, d}}=\frac{Y_{S}}{\rho_{S}}+\frac{\left(1-Y_{S}\right)}{\rho_{L}}$

where $\rho_{L}$ is the liquid density.

Equation 7 can be rewritten in terms of the initial droplet radius, $r_{0, d}$, the final grain radius, $r_{G}$, and the core radius, $r_{C}$ :

$\phi_{\text {Shell }}=\frac{r_{0, d}^{3} \cdot Y_{S} \cdot \rho_{0, d} / \rho_{S}}{r_{G}^{3}-r_{C}^{3}}$

From equation 9, both the core radius and hence the shell thickness, $s$, can be calculated: 


$$
\begin{aligned}
& r_{C}=\left(r_{G}^{3}-\frac{r_{0, d}^{3} \cdot Y_{S} \cdot \rho_{0, d} / \rho_{S}}{\phi_{\text {Shell }}}\right)^{1 / 3} \\
& s=r_{G}-r_{C}
\end{aligned}
$$

In these equations the droplet and grain radius are obtained from the image processing of the video recorded for each test that was performed (Table 1). Figure 4 shows the scheme of a dried grain and its main dimensions that were used in the calculations.

The dried grains were collected and the internal microstructure was observed by Scattering Electron Microscopy. Figure 5 shows the internal structure and the arrangement of the particles in the shell of some of the grains obtained from the drying of droplets under different experimental conditions. The hollow structure can be observed in all cases and the shell thickness was measured. In these images it can also be observed that the sodium chloride always crystallizes at the surface of the grain and, hence, the crystals formed during the evaporation of the liquid do not affect the packing of the particles inside the shell.

Results obtained from equation 11 for the shell thickness were plotted against the ones measured from the micrographs. Figure 6 shows the fitting of the data, in which a very good agreement between theoretical and experimental results can be observed.

Therefore, the packing of the particles in the shell can be said to be constant and equal to the maximum packing that particles can achieve, i.e. RCP. The packing fraction is independent of the solid content, the drying temperature, the $\mathrm{pH}$ of the suspension, the salt content and the initial droplet volume. This packing fraction can be obtained by measuring the viscosity of the suspension at different solid contents and later fitting the data to the Krieger-Dougherty and Quemada equations.

According to equations 10 and 11, once the packing of the particles has been proved to be constant for a particular material, the shell thickness and the degree of hollowness of the grains can be assumed to depend only on the solid content, the initial droplet volume and the final grain diameter, which was shown by the ANOVA analysis to also depend on these two variables.

The final grain diameter can easily be obtained from the drying curves. This variable is related to the critical moisture content, $X_{c r}$, by the following equation [40]: 


$$
d_{G}=d_{c r}=d_{0, d} \cdot \sqrt[3]{\frac{\rho_{L}+X_{c r} \cdot \rho_{S}}{\rho_{L}+X_{0} \cdot \rho_{S}}}
$$

where $d_{c r}$ is the critical diameter equal to the final grain diameter (the shrinkage of the droplet stops at the critical moisture content), $d_{0, d}$ is the initial droplet diameter, $X_{0}$ is the initial moisture content and $\rho_{L}$ and $\rho_{S}$ are the densities of the liquid and the solid respectively.

That critical moisture content, $X_{c r}$, represents the beginning of the second drying period and can be obtained from the drying kinetics of a single droplet. From the drying test, and the evolution of the droplet diameter and position recorded and processed, the critical moisture content was obtained for each experimental condition. The final grain diameter was calculated by means of equation 12. Figure 7 shows the good agreement between the theoretical values and the experimental values obtained from the image processing (Table 1). This means that from the drying kinetics, the final grain diameter and the shell thickness can be predicted for a particular system. Figure 8 summarizes the procedure for calculating the degree of hollowness of grains.

\section{Conclusions}

Single droplets of silica-water nanofluids were dried under different experimental conditions of solid content, $\mathrm{pH}$ value, salt concentration, drying temperature and droplet volume.

The influence of these variables on the final grain diameter, the shell thickness and the mechanical strength of the grain was studied. The solid content and the initial droplet volume were found to influence the final grain diameter and the shell thickness in a positive way. The mechanical strength of the grains was found to depend on the shell thickness (and therefore on the solid content and the droplet volume) and on the $p H$ of the nanofluid. Alkaline conditions increase the "solubility" of silica nanoparticles in water, leading to the formation of highly resistant necks between them.

The packing of particles inside the droplet was proved to be constant for a particular system and equal to RCP. This packing can be obtained from the modelling of the viscosity data using the Quemada equation. For nanoparticles, the value of the packing fraction is lower than for hard microparticles due to the presence of cohesive forces that encumber the movement and rearrangement of particles. 
Hence, the degree of hollowness only depends on the solid content and the final grain diameter. A procedure for predicting the degree of hollowness of the grains from the drying curves was provided. Experimental and theoretical results for the final grain diameter and the shell thickness show good agreement.

\section{Acknowledgments}

R. Mondragón thanks the Spanish Ministry of Education for a pre-doctoral fellowship (FPU program, Ref. AP2008-01077).

\section{References}

[1] Masters K. Spray drying handbook. Longman Scientific and Technical; 1991.

[2] Kastner O, Brenn G, Rensink D, Tropea C. The acoustic tube levitator: a novel device for determining the drying kinetics of single droplets. Chem Eng Technol 2001; 24:3359.

[3] Mezhericher M, Levy A, Borde I. Theoretical models of single droplet drying kinetics: a review. Drying Technol 2010; 28: 278-293.

[4] Hadinoto K, Phanapavudhikul P, Kewu Z, Tan RBH. Novel formulation of large hollow nanoparticles aggregates as potential carriers in inhaled delivery of nanoparticle drugs. Ind Eng Chem Res 2006; 45: 3697-3706.

[5] Hadinoto K, Cheow WS. Hollow spherical nanoparticulate aggregates as potential ultrasound contrast agent: shell thickness characterization. Drug Dev Ind Pharm 2009; 35: 1169-1179.

[6] Sen D, Khan A, Bahadur J, Mazumder S, Sapra BK. Use of small-angle neutron scattering to investigate modifications of internal structure in self-assembled grains of nanoparticles synthesized by spray drying. J Colloid Interface Sci 2010; 347: 25-30.

[7] Iskandar F, Gradon L, Okuyama K. Control of the morphology of nanostrcutured particles prepared by the spray drying of a nanoparticle sol. J Colloid Interface Sci 2003; 265: 296-303.

[8] Sen D, Mazumder S, Melo JS, Khan A, Bhattyacharya S, D’Souza DF. Evaporation driven self-assembly of a colloidal dispersion during spray drying: volume fraction dependent morphological transition. Langmuir 2009; 25: 6690-5.

[9] Bahadur J, Sen D, Mazumder S, Paul B, Khan A, Ghosh G. Evaporation-induced self assembly of nanoparticles in non-buckling regime: volume fraction dependent packing. J Colloid Interface Sci 2010; 351: 357-364.

[10] Shapiro AP, Probstein RF, Random packings of spheres and fluidity limits of monodispere and bidisperse suspensions. Phys Rev Lett 1992; 68 (9): 1422-5. 
[11] Dong KJ, Yang RY, Zou RP, Yu AB. Role of interparticle forces in the formation of random loose packing. Phys Rev Lett 2006; 145505.

[12] German RM. Particle Packing Characteristics. Metal Powder Insdustries Federation. Princeton, New Jersey; 1989.

[13] Yu AB, Feng FL, Zou RP, Yang RY. On the relantionship between porosity and interparticle forces. Powder Technology 2003; 130: 70-6.

[14] Forsyth AJ, Hutton SR, Osborne CF, Rhodes MJ. Effects of interparticle force on the packing of spherical granular material. Phys Rev Lett 2001; 87(24): 244301.

[15] Feng CL, Yu AB. Quantification of the relationship between porosity and interparticle forces for the packing of wet uniform spheres. J Colloid Interface Sci 2000; 231: 136142.

[16] Xu JQ, Zou RP, Yu AB. Packing structure of cohesive spheres. Physical Review E 2004; 69: 032301.

[17] $\mathrm{Yu} \mathrm{AB}$, Bridgwater J, Burbidge A. On the modelling of the packing of fine particles. Powder Technology 1997; 92: 185-194.

[18] Krieger IM, Dougherty TJ. A mechanism for non-Newtonian flow in suspensions of rigid spheres. Trans Soc Rheol 1959; 3: 137-152.

[19] Quemada D. Rheology of concentrated disperse systems and minimum energy dissipation principle. I. Viscosity-concentration relationship. Rheol Acta 1977; 16: 8294.

[20] Sen D, Spalla O, Tache O, Haltebourg P, Thill A. Slow drying of a spray of nanoparticles dispersion. In situ SAXA investigation. Langmuir 2007; 23: 4296-4302.

[21] Brenn G, Kastner O, Rensink D, Tropea C. Evaporation and drying of multicomponent and multiphase droplets in a tube levitator. Proc of the $15^{\text {th }}$ Annual Conf Liquid Atomiz and Spray Syst (ILASS Europe), Toulouse; 1999.

[22] Yarin AL, Brenn G, Kastner O, Rensink D, Tropea C. Evaporation of acoustically levitated droplets. J Fluid Mech 1999; 399: 151-204.

[23] Kastner O, Brenn G, Rensink D, Tropea C, Yarin AL. Investigation of the drying behaviour of suspension droplets in an acoustic tube levitator. Proc 16th Annual Conf Liquid Atomiz and Spray Syst (ILASS Europe), Darmstadt; 2000.

[24] Kastner O, Brenn G, Rensink D, Tropea C. The acoustic tube levitator: a novel device for determining the drying kinetics of single droplets. Chem Eng Technol 2001; 24: $335-9$.

[25] Yarin AL, Brenn G, Kastner O, Tropea C. Drying of acoustically levitated droplets of liquid-solid suspensions: evaporation and crust formation. Phys Fluids 2002; 14: 22892298.

[26] Zaitone B, Hunsmann S, Castanet G, Damaschke N, Ebert V, Tropea C. Evaporation of acoustically levitated droplets. Proc 10th Int Congress on Liquid Atomiz and Spray Syst, Kyoto; 2006.

[27] Mondragon R, Hernandez L, Julia JE, Jarque JC, Chiva S, Zaitone B, Tropea C. Study of the drying behavior of high load multiphase droplets in an acoustic levitator at high temperature conditions. Chem Eng Sci 2011; 66 (12): 2734-2744. 
[28] Mondragon R, Jarque JC, Julia JE, Hernandez L, Barba, A., 2011. Effect of slurry properties and operational conditions on the structure and properties of porcelain tile granules dried in an acoustic levitator. J Eur Ceram Soc 2012; 32, 59-70.

[29] Omrane A, Santesson S, Alden M, Nilsson S. Laser techniques in acoustically levitated micro droplets. Lab on a Chip - Miniaturisation for Chemistry and Biology 2004; 4 (4); 287-291.

[30] Petzold G, Rojas-Reyna R, Mende M, Schwarz S. Application relevant characterization of aqueous silica nanodispersions. J Disp Sci Technol 2009; 30: 1216-1222.

[31] Mondragon R, Julia JE, Barba A, Jarque JC. Characterization of silica-water nanofluids dispersed with an ultrasound probe: a study of their physical properties and stability. Powder Technol 2012; 224: 138-146.

[32] Maskara A, Smith DM. Agglomeration during the drying of fine silica powders, part II: the role of particle solubility. J Am Ceram Soc 1997; 80: 1715-1722.

[33] Iler RK. The chemistry of silica: solubility, polymerization, colloid and surface properties, and biochemistry. John Wiley \& Sons; 1979.

[34] Quemada D. Rheological modelling of complex fluids. I. The concept of effective volume fraction revised. Eur Phys J Appl Phys 1998; 1: 119-127.

[35] Amoros JL, Berltran V, Sanz V, Jarque JC. Electrokinetic and rheological properties of highly concentrated kaolin dispersions: influence of particle volume fraction and dispersant concentration. Appl Clay Sci 2010; 49: 33-43.

[36] Ponton A, Quemada D, Lafuma F, Neel O. Studies of rheological behaviour of colloidal silica suspensions with interaction potential. Colloids and Surfaces A: Physicoquem and Engin Aspects 1996; 119: 255-9.

[37] Griffin GP, Jurinak JJ. Estimation of activity coefficients from the electrical conductivity of natural aquatic systems and soil extracts. Soil Sci 1973; 116: 26-30.

[38] Rubio-Hernandez FJ, Ayucar-Rubio MF, Velazquez-Navarro JF, Galindo-Rosales FJ. Intrinsec viscosity of $\mathrm{SiO}_{2}, \mathrm{Al}_{2} \mathrm{O}_{3}$ and $\mathrm{TiO}_{2}$ aqueous suspensions. $\mathrm{J}$ Colloid Interface Sci 2006; 298, 967-972.

[39] Anoop KB, Kabelac S, Sundararajan T, Das SK. Rheological and flow characteristics of nanofluidos: influence of electroviscous effects and particle agglomeration. J Appl Phys 2009; 106: 034909.

[40] Mezhericher M, Levy A, Borde I. Theoretical drying model of single droplets containing insoluble or dissolved solids. Drying Technol 2007; 25: 1035-1042.

[41] Yarin AL, Weiss DA, Brenn G, Rensink D. Acoustically levitated drops: drop oscillation and break-up driven by ultrasound modulation. Int J Multiphase Flow 2002; 28: 887-910.

[42] Kawahara N, Yarin AL, Brenn G, Kastner O, Durst F. Effect of acoustic streaming on the mass transfer from a sublimating sphere. Phys Fluids 2000; 12: 912-923. 


\section{Figure captions}

Figure 1. Single and cross effects for a) grain diameter, b) shell thickness and c) force.

Figure 2. Evolution of the mechanical strength with the shell thickness. Influence of $p H$.

Figure 3. Evolution of relative viscosity with effective volume fraction and $p H$. Experimental data and modelled curve.

Figure 4. Internal microstructure of a dried grain and main dimensions.

Figure 5. Internal microstructure of dried grains at different drying conditions.

Figure 6. Shell thickness determined experimentally and theoretically.

Figure 7. Final grain diameter determined experimentally and theoretically.

Figure 8. Procedure to evaluate the degree of hollowness of grains. 


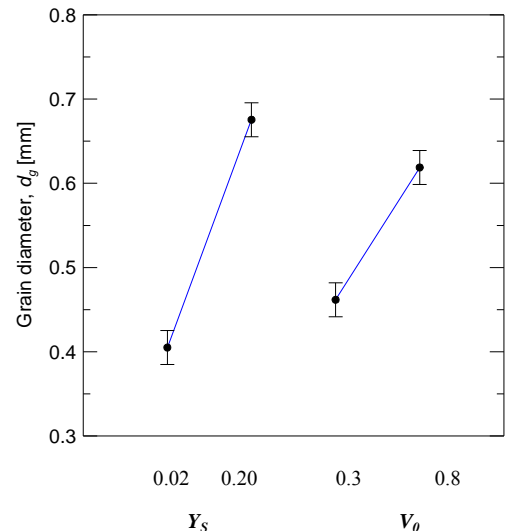

a)

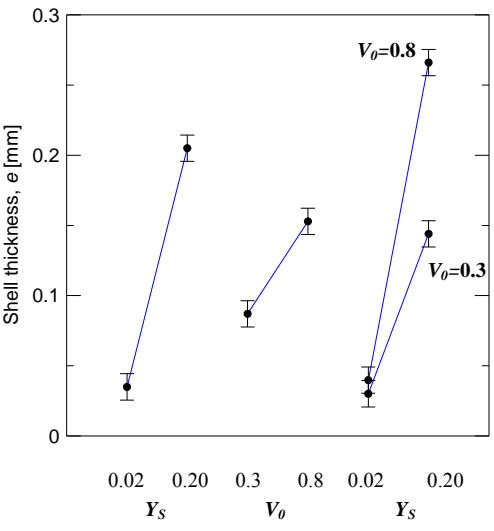

b)

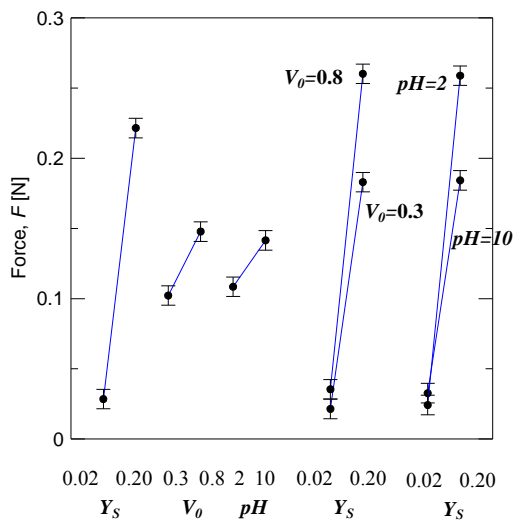

c)

Figure 1. Single and cross effects for a) grain diameter, b) shell thickness and c) force. 


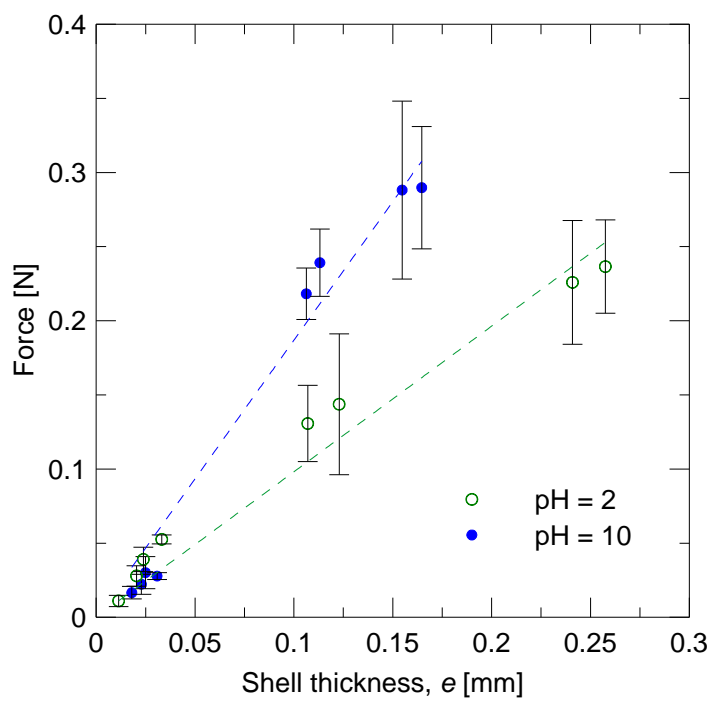

Figure 2. Evolution of the mechanical strength with the shell thickness. Influence of $p H$. 


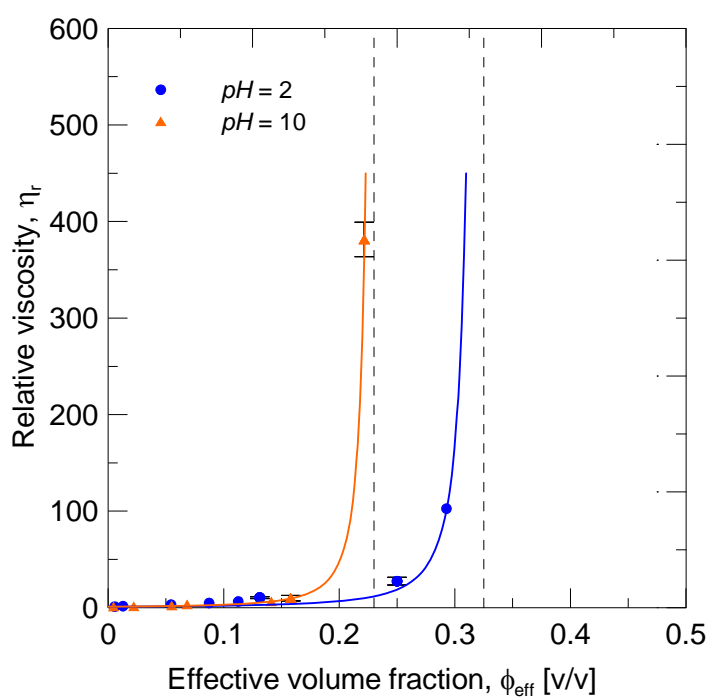

Figure 3. Evolution of relative viscosity with effective volume fraction and $p H$. Experimental data and modelled curve. 


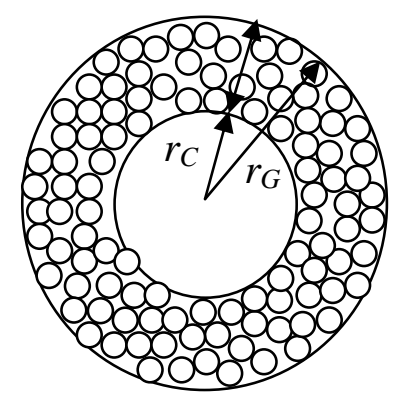

Figure 4. Internal microstructure of a dried grain and main dimensions. 

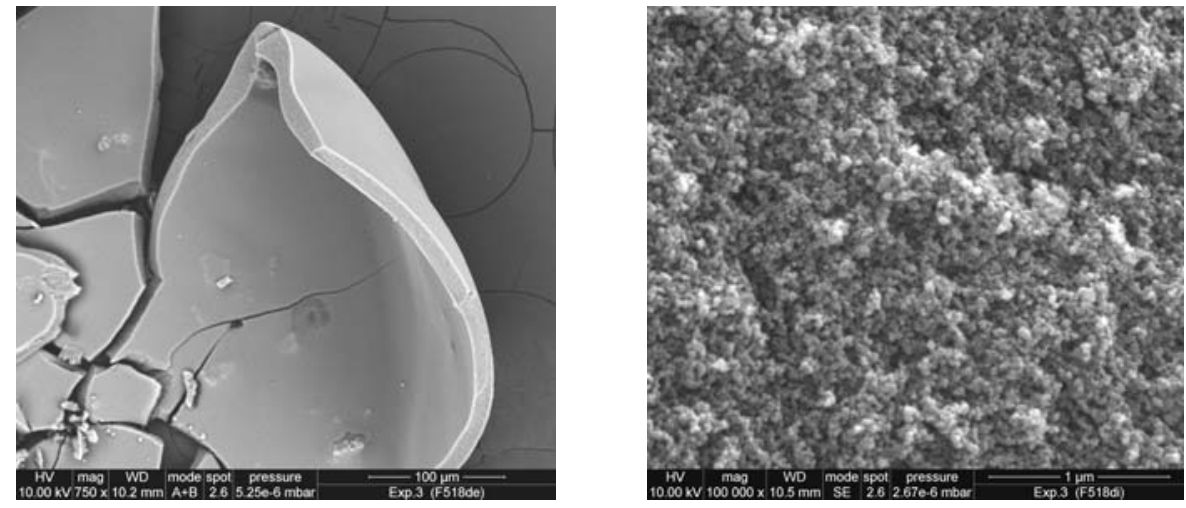

$Y_{S}=0.02, p H=10,[\mathrm{NaCl}]=0, T=80^{\circ} \mathrm{C}, V_{0}=0.3 \mu \mathrm{l}$
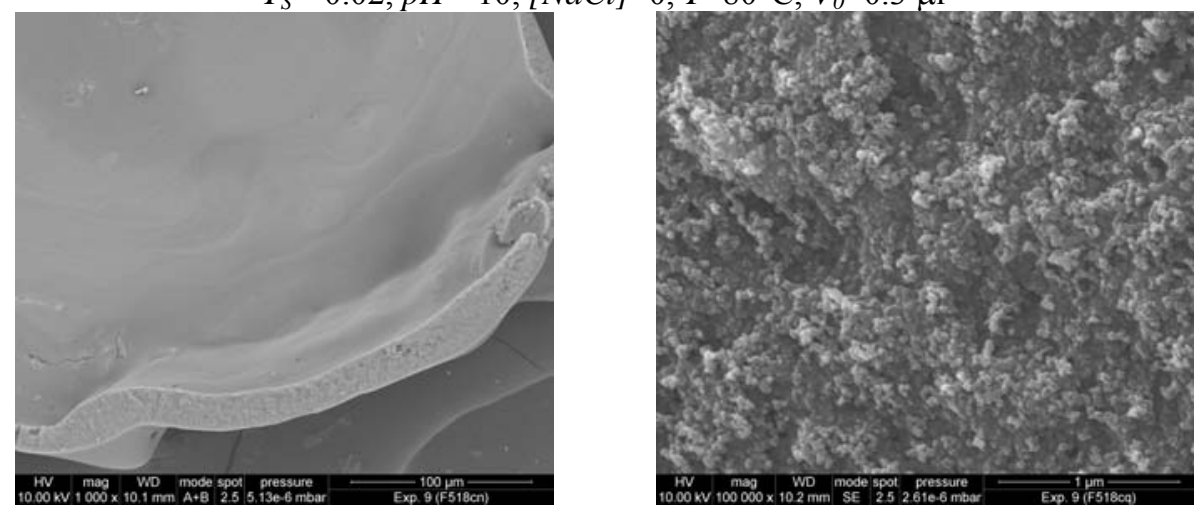

$Y_{S}=0.02, p H=2,[\mathrm{NaCl}]=0, T=120^{\circ} \mathrm{C}, V_{0}=0.3 \mu \mathrm{l}$
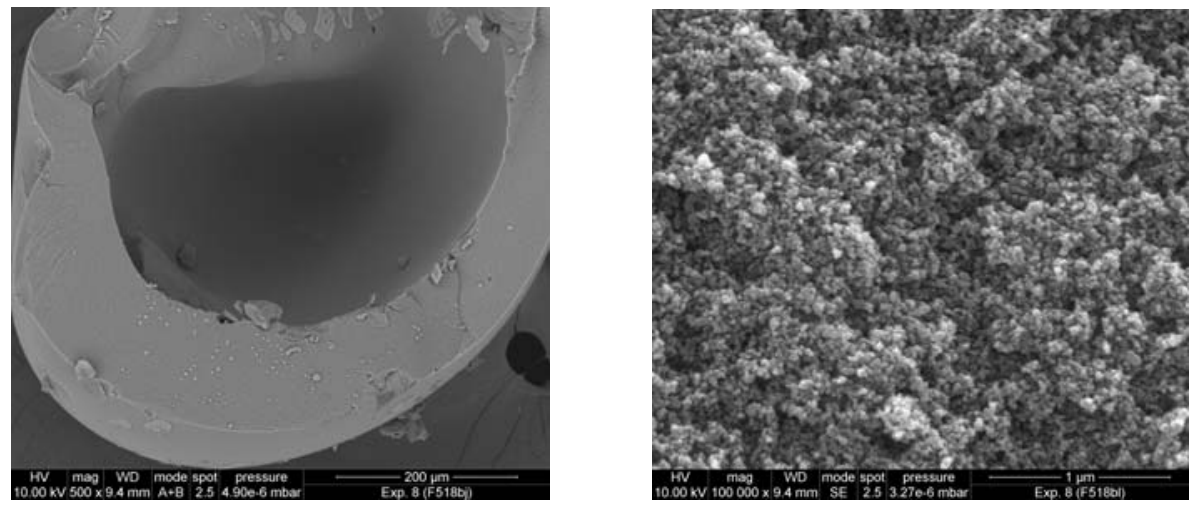

$Y_{S}=0.20, p H=10,[\mathrm{NaCl}]=0.05 \mathrm{M}, T=80^{\circ} \mathrm{C}, V_{0}=0.3 \mu \mathrm{l}$
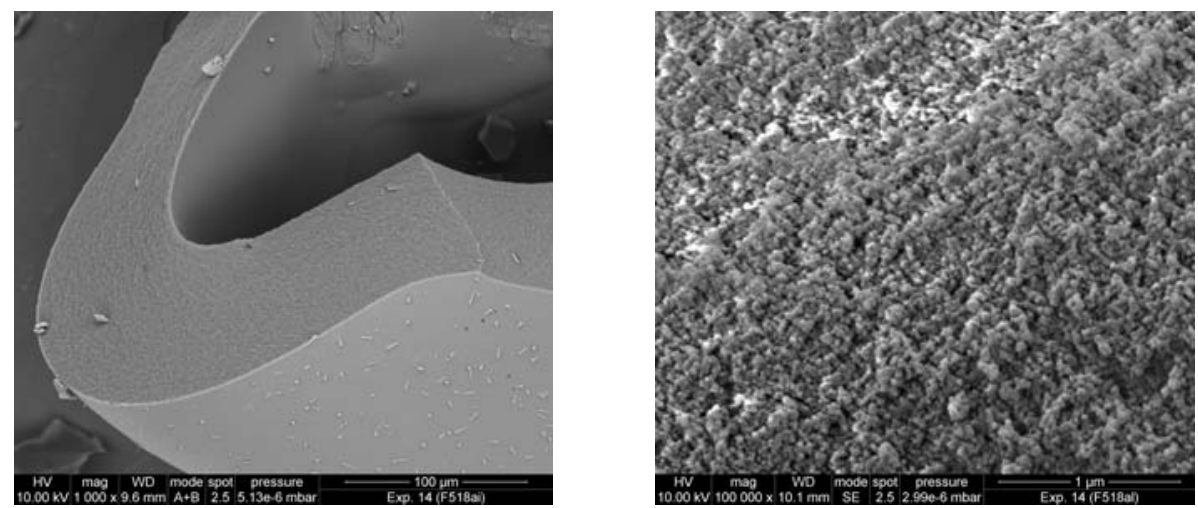

$Y_{S}=0.20, p H=2,[\mathrm{NaCl}]=0.05 \mathrm{M}, T=120^{\circ} \mathrm{C}, V_{0}=0.3 \mu \mathrm{l}$

Figure 5. Internal microstructure of dried grains at different drying conditions. 


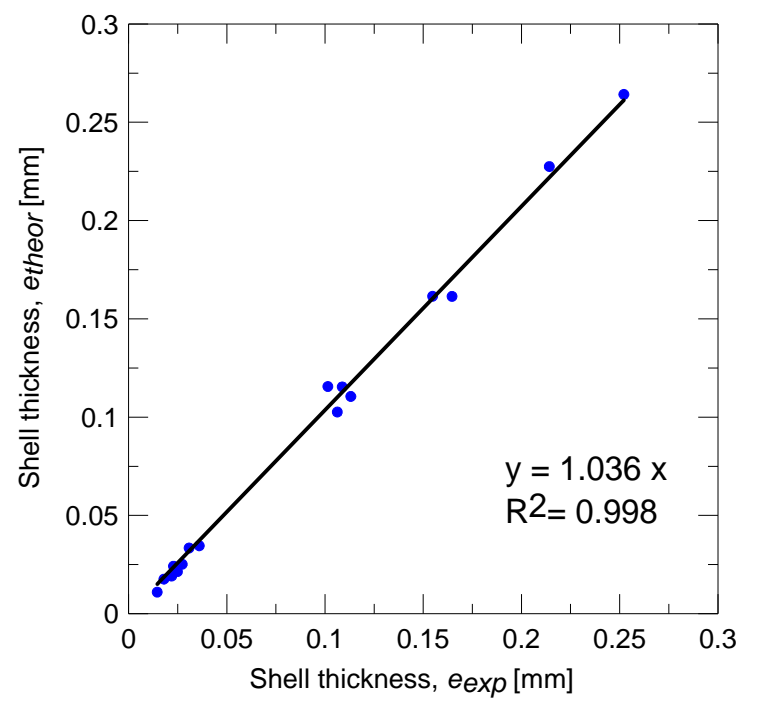

Figure 6. Shell thickness determined experimentally and theoretically. 


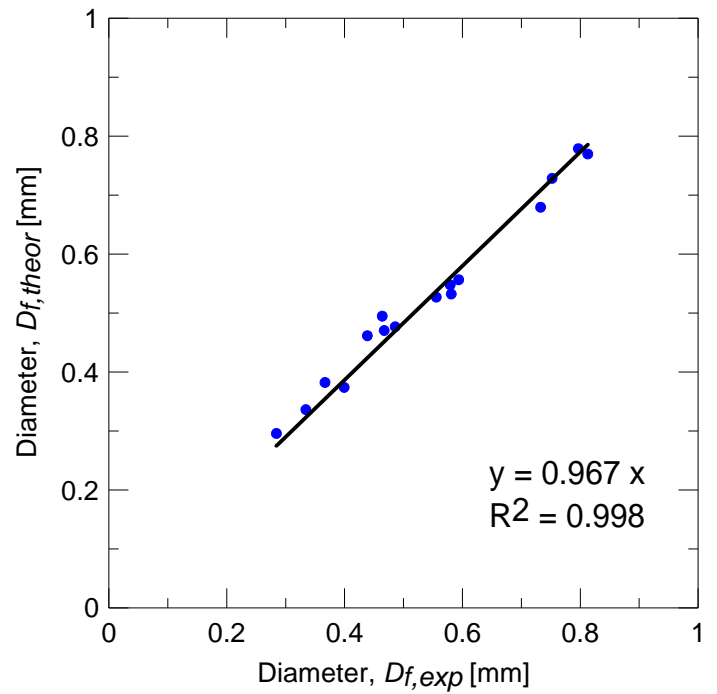

Figure 7. Final grain diameter determined experimentally and theoretically. 


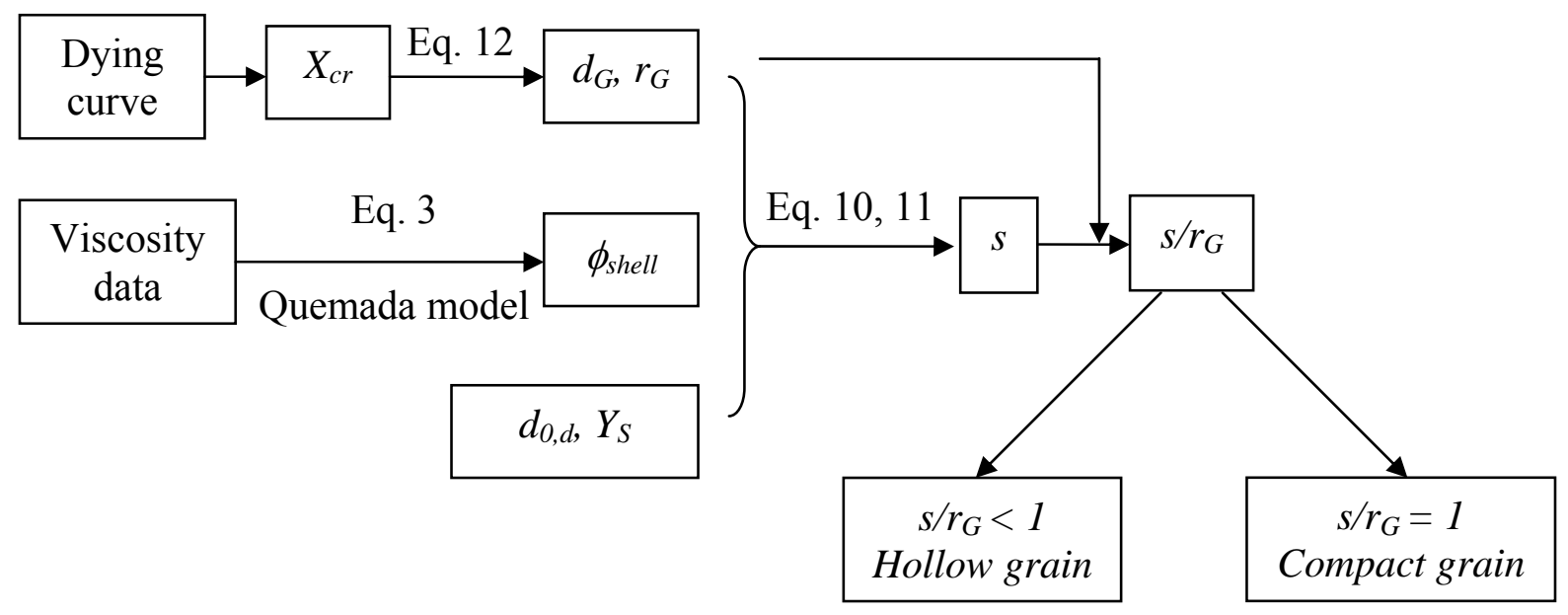

Figure 8. Procedure to evaluate the degree of hollowness of grains. 


\section{Table captions.}

Table 1. Experimental matrix (DoE) and results.

Table 2. Probability of the F-distribution. 
Table 1. Experimental matrix (DoE) and results.

\begin{tabular}{ccccccccc}
\hline \hline Exp & $\begin{array}{c}\boldsymbol{Y}_{\boldsymbol{S}} \\
{[\mathbf{w} / \mathbf{w}]}\end{array}$ & $\boldsymbol{p H}$ & $\begin{array}{c}{[\mathbf{N a C l}]} \\
{[\mathbf{M}]}\end{array}$ & $\begin{array}{c}\boldsymbol{T} \\
{\left[{ }^{\mathbf{0}} \mathbf{C}\right]}\end{array}$ & $\begin{array}{c}\boldsymbol{V}_{\boldsymbol{o}} \\
{[\boldsymbol{\mu} \mathbf{l}]}\end{array}$ & $\begin{array}{c}\boldsymbol{d}_{\mathbf{G}} \\
{[\mathbf{m m}]}\end{array}$ & $\begin{array}{c}\boldsymbol{e} \\
{[\mathbf{m m}]}\end{array}$ & $\begin{array}{c}\boldsymbol{F} \\
{[\mathbf{N}]}\end{array}$ \\
\hline 1 & 0.02 & 2 & 0 & 80 & 0.8 & 0.467 & 0.027 & 0.039 \\
\hline 2 & 0.20 & 2 & 0 & 80 & 0.3 & 0.581 & 0.101 & 0.131 \\
\hline 3 & 0.02 & 10 & 0 & 80 & 0.3 & 0.284 & 0.025 & 0.030 \\
\hline 4 & 0.20 & 10 & 0 & 80 & 0.8 & 0.813 & 0.165 & 0.290 \\
\hline 5 & 0.02 & 2 & 0.05 & 80 & 0.3 & 0.334 & 0.022 & 0.028 \\
\hline 6 & 0.20 & 2 & 0.05 & 80 & 0.8 & 0.733 & 0.214 & 0.226 \\
\hline 7 & 0.02 & 10 & 0.05 & 80 & 0.8 & 0.439 & 0.031 & 0.028 \\
\hline 8 & 0.20 & 10 & 0.05 & 80 & 0.3 & 0.579 & 0.106 & 0.218 \\
\hline 9 & 0.02 & 2 & 0 & 120 & 0.3 & 0.399 & 0.015 & 0.011 \\
\hline 10 & 0.20 & 2 & 0 & 120 & 0.8 & 0.752 & 0.252 & 0.237 \\
\hline 11 & 0.02 & 10 & 0 & 120 & 0.8 & 0.486 & 0.023 & 0.022 \\
\hline 12 & 0.20 & 10 & 0 & 120 & 0.3 & 0.594 & 0.113 & 0.239 \\
\hline 13 & 0.02 & 2 & 0.05 & 120 & 0.8 & 0.464 & 0.036 & 0.053 \\
\hline 14 & 0.20 & 2 & 0.05 & 120 & 0.3 & 0.556 & 0.109 & 0.144 \\
\hline 15 & 0.02 & 10 & 0.05 & 120 & 0.3 & 0.367 & 0.018 & 0.017 \\
\hline 16 & 0.20 & 10 & 0.05 & 120 & 0.8 & 0.797 & 0.155 & 0.288 \\
\hline \hline
\end{tabular}


Table 2. Probability of the F-distribution.

\begin{tabular}{cccc}
\hline $\begin{array}{c}\text { Input } \\
\text { variable }\end{array}$ & $\boldsymbol{d}_{\mathbf{G}}$ & $\boldsymbol{e}$ & $\boldsymbol{F}$ \\
\hline \hline$Y_{S}$ & 0.0000 & 0.0000 & 0.0000 \\
\hline$p H$ & 0.6605 & 0.0671 & 0.0014 \\
\hline$[\mathrm{NaCl}]$ & 0.5220 & 0.9793 & 0.9694 \\
\hline$T$ & 0.2780 & 0.0947 & 0.7186 \\
\hline$V_{0}$ & 0.000 & 0.0001 & 0.0002 \\
\hline$Y_{S} \times V_{0}$ & - & 0.0002 & 0.0019 \\
\hline$Y_{S} \times p H$ & - & - & 0.0003 \\
\hline \hline
\end{tabular}

